\title{
Salud mental en situaciones de desastres
}

Deborah León-Amenero 1, 2, a; Jeff Huarcaya-Victoria* 3, 4, b

RESUMEN

Los desastres son eventos a gran escala, muchas veces inesperados. Usualmente, se suele prestar más atención a las consecuencias materiales y físicas de los mismos. Sin embargo, las intervenciones en estas situaciones están incompletas si no se abordan aspectos de salud mental. El propósito del presente artículo es realizar una revisión de las consecuencias psicosociales asociadas a un desastre, entre ellas, problemas de salud mental, como el trastorno de estrés postraumático, trastornos depresivos, consumo de sustancias psicoactivas, etc., y las consecuencias psicosociales en la estructura familiar; asimismo, resaltar los posibles factores de riesgo asociados a su presentación. Las estrategias de intervención son variadas, y dependen del tiempo, la persona y el nivel de acción. Es necesario que, como país, tengamos diversas estrategias y modelos de intervención para intentar atenuar el impacto psicosocial del desastre. Sin embargo, todavía persisten barreras en el manejo de la salud mental, por lo que se hace necesario plantear posibles direcciones futuras a fin de lograr un menor impacto en la sociedad y una recuperación más temprana.

Palabras clave: Desastres; Salud mental; Perú; Trastornos por estrés postraumático; Depresión (Fuente: DeCS BIREME).

\section{Mental health in disaster situations}

\section{ABSTRACT}

Disasters are large-scale events which are often unexpected. Usually more attention is paid to the material and physical consequences of disasters. However, interventions in disaster situations are incomplete if mental health issues are not addressed. This work aims to review the psychosocial consequences associated with a disaster, including mental health problems such as post-traumatic stress disorders, depressive disorders, psychoactive substance abuse, etc., and the psychosocial consequences in the family structure, highlighting the possible risk factors related to their presentation. The intervention strategies are varied and depend on the time, the person and the level of action. It is necessary that we, as a country, have different intervention strategies and models to try to mitigate the psychosocial impact of disasters. Nevertheless, there continue to be barriers in the management of mental health, so it is necessary to consider possible future directions in order to achieve a lower impact on society and an earlier recovery.

Keywords: Disasters; Mental health; Peru; Stress disorders, post-traumatic; Depression (Source: MeSH NLM).

1. Hospital Nacional Guillermo Almenara Irigoyen, Servicio de Psiquiatría Infanto-Juvenil. Lima, Perú.

2. Universidad Nacional Mayor de San Marcos, Facultad de Medicina de San Fernando. Lima, Perú.

3. Universidad de San Martín de Porres, Facultad de Medicina, Centro de Investigación en Salud Pública. Lima, Perú.

4. Hospital Nacional de la Policía Nacional del Perú Luis N. Sáenz, Departamento de Psiquiatría. Lima, Perú.

a. Médico Residente de Psiquiatría Infanto-juvenil.

b. Médico Psiquiatra.

* Autor corresponsal. 


\section{INTRODUCCIÓN}

El Perú es un país expuesto a diferentes situaciones de desastres que pueden ser naturales (el fenómeno El Niño), producidos por el hombre (aniegos), sociales (terrorismo), etc. Un desastre es un evento a gran escala, usualmente inesperado. Se postula que, en promedio, 150 millones de personas son afectadas anualmente por un desastre. De todos ellos, aproximadamente el $33 \%$ desarrollarían un trastorno de estrés postraumático (TEPT) y el $20 \%$ algún otro trastorno de salud mental comórbido ${ }^{(1)}$.

Son diversos los motivos por los cuales un desastre puede ser considerado como un problema de salud pública, por ejemplo: 1) la gran demanda de atención necesaria debido a las muertes, lesiones y enfermedades que ocasionan, 2) alteración de la infraestructura de servicios básicos como agua, luz, desagüe y comunicaciones, 3) el incremento del riesgo de enfermedades transmisibles, y 4) las consecuencias psicosociales en las personas afectadas (2). De entre todos estos, las consecuencias psicosociales determinarán el grado de funcionalidad y recuperación de las personas y de la comunidad afectada.

El propósito de este trabajo es revisar las consecuencias psicosociales asociadas a un desastre, entre ellas, problemas de salud mental como el TEPT, trastornos depresivos, consumo de sustancias psicoactivas, etc., y resaltar los posibles factores de riesgo asociados a su presentación. Dado que un desastre se considera como un proceso, se plantearán posibles factores asociados antes, durante y después del desastre.

\section{DESASTRE}

Etimológicamente, la palabra desastre se encuentra formada por el sufijo dis- (separación, oposición, contrariedad) y la palabra astrum (astro, estrella) ${ }^{(3)}$. En un inicio, se empleaba para referirse a la desintegración de las estrellas, lo cual era interpretado como una predicción de catástrofes. Actualmente, su definición gira en torno a la catástrofe en sí.

Para la Organización Mundial de la Salud (OMS), un desastre es: "Un acto de la naturaleza de tal magnitud que da origen a una situación catastrófica en la que súbitamente se desorganizan los patrones cotidianos de vida y la gente se ve hundida en el desespero y sufrimiento. Como resultado de ello las víctimas necesitan víveres, ropa, vivienda, asistencia médica y de enfermería, así como otros elementos fundamentales de vida y protección contra factores y condiciones ambientales desfavorables" (4).
Si bien muchos de los desastres son de origen natural, no se limitan a este grupo. De manera general se pueden clasificar en desastres naturales y los ocasionados por el ser humano ${ }^{(4,5)}$ :

1) Desastres naturales: meteorológicos, hidráulicos, topológicos, etc.

2) Desastres ocasionados por el hombre:

a) Desastres generados por el hombre (intencionales o no intencionales): de transporte, biológicos, químicos y tecnológicos.

b) Desastres sociales: terrorismo, hambruna $y$ guerras.

Respecto a sus características, Math et al. (6) plantean que estas son el inicio brusco, impredecible e incontrolable, la destrucción en gran magnitud que ocasiona la pérdida de vidas humanas y el sufrimiento, que exceden la capacidad de afrontamiento de una comunidad.

Villalibre ${ }^{(7)}$, luego de estudiar las distintas definiciones de desastre, encontró que las principales características que presentan son las siguientes: 1) ocasionan daños en estructuras sociales, 2) ocasionan daños materiales y humanos, 3) tiene consecuencias psicológicas, y 4) no hay recursos suficientes para afrontarlo. De esta manera, no considera que los desastres, necesariamente, deban presentarse de manera brusca ni impredecible. Si bien Villalibre ${ }^{(7)}$ considera que el desastre debe cumplir con la característica de "evento" o "suceso", para Calderón (4), un desastre es un proceso, lo que le da una dimensión temporal, que tiene un "antes, durante y después". Para la definición de un desastre, más que las causas que lo ocasionan, son las consecuencias las que terminan de dar valor a su significado, $y$, entre ellas, son las consecuencias psicológicas las que muchas veces determinarán la gravedad según la capacidad de recuperación de la población.

En el Perú, el desastre natural característico es el fenómeno de patrón climático conocido como El Niño que causa inundaciones devastadoras, los más notables han ocurrido en los años años 1982-1983 y 1997-1998. El último El Niño, en el año 2017, ocasionó un aproximado de 1138619 personas afectadas, de las cuales fueron víctimas 235806 y fallecieron $145{ }^{\text {(8) }}$. Dentro de los desastres sociales encontramos el conflicto armado interno del Perú que se desarrolló durante las décadas de 1980 a 2000. La Comisión de la Verdad y Reconciliación, en el año 2003, determinó que el número de personas muertas y desaparecidas, tanto víctimas de los grupos subversivos terroristas como de las propias Fuerzas Militares del Perú, durante este conflicto armado, ascendió a 69280 personas ${ }^{(9)}$. 


\section{IMPACTO PSICOSOCIAL DE LOS DESASTRES}

Un desastre puede alterar el significado y hasta el propósito de la vida de quienes lo experimentan; incluso pueden llegar a modificar el punto de vista que alguien tenga respecto al mundo y a sí mismo ${ }^{(10)}$.

\section{Respuesta al desastre}

La respuesta más frecuente es la de un duelo normal, caracterizado por ser directamente proporcional a la magnitud de la pérdida/desastre, el cual se resuelve en los meses posteriores. Usualmente, la duración es menor de 6 a 12 meses de ocurrido el evento. Entre los posibles pensamientos o conductas que podría presentar una persona están la culpa, el miedo a perder el control de las emociones y el consumo de sustancias. A pesar de no tratarse de un duelo patológico, es necesario considerar a estas personas en riesgo de desarrollar, eventualmente, un trastorno mental, por lo que requieren cierto seguimiento ${ }^{(6)}$

En general, como es de esperarse, existe una variabilidad humana en respuesta a los desastres; mientras que la mayoría de personas lograrán recuperarse, una minoría tendrá mayores dificultades en lograr un funcionamiento similar al previo, y otro porcentaje no logrará recuperarse, incluso luego de haber recibido ayuda profesional.

Entre algunos factores que se asocian a una respuesta adecuada al desastre están la resistencia (capacidad de soportar los eventos negativos), la resiliencia (capacidad de recuperarse ante la adversidad) y la recuperación (capacidad de retomar el nivel de funcionamiento previo) ${ }^{(11)}$. Otros factores, más generales, que influyen también en el impacto son la edad, que es el riesgo mayor en los menores, y la presencia de familiares que sufran el desastre, ya que la persona no solo presentará psicopatología asociada a su pérdida, sino también asociada a las pérdidas y padecimientos que sus seres queridos experimenten. Además, también se postula que la variabilidad genética podría influir en la manera en que cada persona afronta un desastre ${ }^{(12)}$.

Factores que influyen en el impacto psicosocial De acuerdo a Abeldano y Fernández ${ }^{(13)}$, existen algunos factores que determinarán el impacto psicosocial de un desastre:

1) La naturaleza del evento: Las características de un desastre que producen un mayor impacto están el que sea inesperado, que sea ocasionado por el hombre y que provoque una afección colectiva.

Por lo general, el evento que ocurre sin aviso y no permite establecer medidas de prevención es que producirán mayor impacto psicosocial.

2) Ambiente y circunstancias asociadas: Las personas que pertenecen a comunidades más pobres, con limitaciones al acceso a servicios de salud o servicios sociales son las más vulnerables.

Como ya mencionamos, un desastre forma parte de un proceso. De esta manera, durante su desarrollo existe un antes, un durante y un después. Goldmann ${ }^{(5)}$, con base en la obra de Klein et al., determinó algunos posibles factores de riesgo asociados al desarrollo de un trastorno mental según el momento del desastre (Figura 1).

\section{Consecuencias en la salud mental}

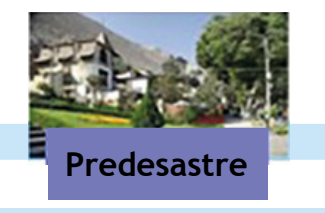

Trastorno mental previo : TEPT. trastorno depresivo transtorno de conducta por consumo de sustancias.

Mujeres : TEPT. trastorno depresivo.

Hombres : Consumo de sustancias.

Población infantil : ansiedad y depresión.

Población pobre, minorías étnicas, solteros, tener hijos, haber sido participes de un desastre previo, pobre apoyo social.

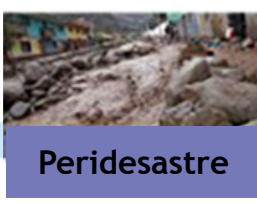

Dosis-respuesta : a mayor severidad del desastre, mayor riesgo de desarrollar un transtorno mental secundario al mismo.

Gravedad : tipo de desastre, duración proximidad. número e intensidad de los desastres similares experimentados por la persona.

Determinar la amenaza de la vida, la presencia de lesiones graves, el haber presenciado la muerte o daño de otra persona.

Figura 1. Factores de riesgo asociados al desarrollo de un trastorno mental según el momento del desastre 
A diferencia de las lesiones físicas, los resultados adversos para la salud mental en las situaciones de desastre no suelen ser evidentes $y$, por lo tanto, se requiere de un enfoque sistemático para la identificación de casos y la selección de las intervenciones apropiadas ${ }^{(14)}$. Entre los principales trastornos psiquiátricos secundarios a un desastre encontramos:

1) TEPT: Es uno de los principales trastornos asociados a un desastre, puede presentarse hasta en el $30-40 \%$ de todas las víctimas directas del desastre, en el 10-20\% de los trabajadores de rescate, y 5-10\% en las víctimas indirectas ${ }^{(15-17)}$. La evidencia también muestra que las mujeres tienen más probabilidades de experimentar síntomas de TEPT (18). En un reciente metanálisis se encontró que los adultos mayores tendrían hasta 2,11 veces más probabilidades de experimentar síntomas de TEPT cuando se exponen a desastres, en comparación con adultos más jóvenes ${ }^{(19)}$. Debemos buscar en estas personas la presencia de re-experimentación del suceso desastroso, que se puede manifestar en ideas, pesadillas, flashbacks, todo lo cual se acompaña de una evitación del estímulo con alteración de la actividad autonómica. En los estudios que evalúan el TEPT en los desastres, las escalas más utilizadas son el Impact Event Scale (IES) y el Post Traumatic Stress Disordercivilian checklist (PCL-C) ${ }^{(20)}$.

2) Trastorno depresivo: Es el trastorno que se encuentra con más frecuencia se asociado a un desastre luego del TEPT. La gravedad y probabilidad de aparición dependerá de la presencia de un trastorno afectivo previo al desastre. En un estudio se encontró que, en una muestra de 129 personas víctimas del fenómeno El Niño en el 2017, un 10 \% presentaron depresión ${ }^{(21)}$.

3) Trastorno de conducta por el consumo de múltiples sustancias: Si bien es menos frecuente que los dos anteriores, se ha encontrado un incremento en el consumo de sustancias posterior a los desastres, esto probablemente como un mecanismo para evitar afrontar el evento y sus consecuencias.

Consecuencias psicosociales en la familia

Los desastres suelen afectar a la familia más que aun solo individuo. De esta manera, el impacto psicosocial lo vive la familia en conjunto, y se puede manifestar con síntomas de irritabilidad y depresión entre sus miembros, así como el incremento de situaciones conflictivas entre ellos ${ }^{(22)}$.

$\mathrm{Si}$ consideramos que la infancia es un periodo de gran dependencia de otras personas, usualmente de los padres y la familia, se puede deducir que una alteración en el funcionamiento de esta se podría traducir en el posible desarrollo de un TEPT y síntomas depresivos entre los miembros más jóvenes de la misma. Además, la ansiedad de los padres puede llevar, en ocasiones, a emplear estrategias de evitación frente al trauma, con las consecuencias que esto conlleva en sus hijos ${ }^{(23)}$.

Algunas de las características que incrementan la vulnerabilidad de los niños ante un desastre son la escasa comunicación entre los padres e hijos respecto al desastre ocurrido, conflictos entre los hijos con los padres (o con distintos miembros de la familia), alteración del funcionamiento de la familia a partir del desastre, y exposición (no supervisada por los padres) a medios de comunicación que relaten el desastre de manera inadecuada para niños y adolescentes ${ }^{(22,24)}$. Sin embargo, estas características pueden modificarse una vez que son identificadas, y se reduce así la posibilidad de desarrollar algún tipo de psicopatología en la población infanto-juvenil; además de disminuir el riesgo de trastornos de salud mental cuando sean adultos ${ }^{(25)}$.

Existen pocos estudios que valoren el impacto de los desastres en las relaciones matrimoniales y de pareja. En un estudio realizado a 40 mujeres afroamericanas que sobrevivieron al huracán Katrina se investigó, a través de entrevistas, cómo el huracán afectó sus relaciones íntimas ${ }^{(26)}$. Se encontró que, aunque muchas participantes informaron cambios negativos en sus relaciones, otras informaron que estas se fortalecieron. El desastre provocó factores de estrés extremos, incluido el desempleo y las separaciones prolongadas, todo lo cual socavó el funcionamiento individual y relacional. Sin embargo, otras participantes señalaron cambios positivos, como nuevas oportunidades de empleos y altos niveles de comunicación efectiva y apoyo en sus relaciones ${ }^{(26)}$. Estos efectos positivos no solo pueden observarse a nivel familiar, sino también a nivel de la comunidad, en la cual puede existir una mayor cohesión, tal como vimos en el último fenómeno de El Niño en el Perú (27).

\section{INTERVENCIONES EN SALUD MENTAL ASOCIADAS A DESASTRES}

Modelos de intervención

Intervención centrada en el tiempo

Si recordamos que un desastre es un proceso, este modelo de intervención hace énfasis en el momento de la intervención que puede ser antes, durante o después del desastre. En ese sentido, si bien la mayor parte de 
los esfuerzos aún están dirigidos a la etapa "durante el desastre" , últimamente, se han planteado estrategias de prevención, promoción y recuperación, que están dirigidas al "antes" y al "después" del desastre (13).

Bambarén (2) nos presenta cuatro fases asociadas a este modelo de intervención:

1) Fase precrítica: dado a horas o días previos al desastre, busca prevenir o atenuar los daños que se producirían a causa del evento.

2) Fase crítica: incluye el desastre y hasta 72 horas después de ocurrido este. En esta fase se busca proteger la integridad física, así como satisfacer las necesidades básicas de las personas, evitar la desorganización social y brindar la primera ayuda psicológica.

3) Fase postcrítica: periodo que incluye hasta los 6 primeros meses posteriores al desastre. En este momento se busca apoyar en la organización de la comunidad para lograr una coordinación interinstitucional, se trabaja especialmente con poblaciones vulnerables, y se desarrollan intervenciones en crisis. La atención psicosocial en este punto es de suma importancia.

4) Fase de recuperación: luego de los 6 primeros meses de ocurrido el desastre. A partir de este punto se busca el desarrollo de proyectos ocupacionales, la continuidad de la organización comunitaria y la coordinación interinstitucional.

Por otra parte, es muy importante tener en cuenta la rapidez en el inicio de la atención. En los últimos años se ha trabajado cada vez más temprano en el abordaje del trauma secundario a un desastre. La acción ya no se basa en el manejo de las personas que acuden a evaluación luego de presentar síntomas de TEPT o algún otro trastorno; actualmente, la atención se brinda lo más inmediatamente posible, a fin de evitar, o minimizar, las consecuencias de un posible trastorno mental.

Un ejemplo de esto lo encontramos en el Perú durante el año 2017. Ante el fenómeno de El Niño se declararon en Emergencia Sanitaria los departamentos de Tumbes, Cajamarca, Ancash, La Libertad, Piura, Lambayeque y Lima provincias. Para ello, en las primeras semanas luego de ocurrido los desastres se reunieron miembros del Ministerio de Salud (MINSA), la OPS/OMS, Fundación Manantial, entre otros, con la finalidad de poder brindar atención de salud lo más pronto posible a las poblaciones damnificadas (28). En aquella oportunidad se hizo especial énfasis en la salud mental de los damnificados.

Intervención según el nivel de acción

Este modelo está enfocado en brindan atención según las necesidades de la población afectada, ya no en lo sobrevivientes ni en la recuperación de bienes. Un punto importante en este modelo es la capacidad de reunir a todos los afectados, a la comunidad y a distintos actores sociales a fin de poder resolver las necesidades que se identifiquen.

El Sphere Proyect (1997) es un claro ejemplo de este tipo de intervención. Este proyecto ha implementado estrategias integrales en casos de desastres y tiene como objetivo "mejorar la calidad de la asistencia humanitaria prestada a personas afectadas por los desastres, y aumentar el grado de rendición de cuentas del sistema humanitario en la respuesta en casos de desastres" (29). En él se desarrolló la Carta Humanitaria, la cual muestra los principios en los cuales se rige la acción humanitaria y reafirma el derecho de las personas afectadas a recibir protección y asistencia; además, se plantean las normas mínimas en cinco materias distintas: 1) comunes a todos los sectores, 2) abastecimiento de agua, saneamiento y fomento de la higiene, 3) seguridad alimentaria, nutrición y ayuda alimentaria, y 4) refugios, asentamientos y artículos no alimentarios y 5) servicios de salud.

Intervención centrada en la persona

De acuerdo a este modelo, se busca el empoderamiento y la participación de toda la comunidad afectada para que se integre en la labor asistencial. De acuerdo a Abenaldo y Fernández ${ }^{(13)}$, la idea es centrarse en el potencial de la comunidad en lugar de sus deficiencias, tanto a nivel familiar como comunitario en sí.

\section{Barreras en el manejo de la Salud Mental}

A pesar de los distintos modelos de atención existentes, tanto en el Perú como en el mundo, aún no se logra prevenir el desarrollo de patologías como el TEPT, trastornos depresivos, entre otros, en las personas que han sido parte de un desastre.

De acuerdo a lo postulado por Kazlauskas (30), existen barreras que no permiten brindar una atención adecuada que evite el desarrollo de distintos trastornos, las cuales son:

1) Falta de reconocimiento social del trauma en los sobrevivientes: En ocasiones los sobrevivientes no suelen tener el reconocimiento ni recibir los cuidados que merecen, que son destinados a quienes 
se considera de mayor gravedad. A esto, se le suma el que algunos sobrevivientes evitan buscar la ayuda necesaria.

2) Evitación del trauma: A fin de no revivir la situación traumática, algunas personas pueden finalmente, evitar recibir la atención psiquiátrica.

3) Recursos limitados: El tratamiento más adecuado para el manejo del TEPT suelen ser las intervenciones psicoterapéuticas; sin embargo, no suelen ser ofrecidas en suficientes instituciones.

4) Conflictos y desastres en curso: El manejo del TEPT o de distintos trastornos mentales no se puede dar de manera adecuada si es que la persona vivirá otro desastre pronto.

¿Qué hacer? Debemos aprender de experiencias pasadas. Por ejemplo, en la 13th European Society for Traumatic Stress Studies ${ }^{(12)}$ se plantearon ocho “lecciones aprendidas" de 6 de los desastres más importantes ocurridos en los últimos 10 años (Tabla 1).

Existen algunas posibles alternativas de investigación a fin de atenuar las barreras descritas ${ }^{(30)}$ :

1) Sensibilidad cultural: Determinar si es que existen diferencias culturales en el desarrollo y pronóstico de los trastornos de salud mental secundarios a los desastres, a fin de lograr un mejor abordaje según cada población.

2) Modelos de atención de la salud: Considerar modelos alternativos, por ejemplo, las historias clínicas electrónicas.

3) Participación de voluntarios no profesionales: Con la finalidad de conseguir una mayor cobertura y prontitud en la atención.

Tabla 1. “Lecciones aprendidas" de 6 de los desastres más importantes ocurridos en los últimos 10 años (12)

\begin{tabular}{|l|l}
\hline & Adaptar la respuesta psicosocial al desastre específico. \\
& Brindar atención psicosocial multidimensional. \\
& Focalizar grupos de población en riesgo. \\
Abordar proactivamente las barreras en el acceso a la atención. \\
$\begin{array}{l}\text { Lecciones } \\
\text { aprendidas }\end{array}$ & $\begin{array}{l}\text { Reconocer las dimensiones sociales y fuentes de resiliencia. } \\
\text { Extender los roles para los profesionales de la salud mental. }\end{array}$ \\
& $\begin{array}{l}\text { Coordinar e integrar de manera eficiente los servicios de respuesta ante } \\
\text { los desastres. } \\
\text { Integrar la investigación y evaluación en la planificación de respuesta } \\
\text { ante los desastres. }\end{array}$
\end{tabular}

Las duras lecciones de los desastres en el Perú, como el terremoto en Pisco en 2007 o el reciente fenómeno de El Niño en 2017, ayudan a entender la salud mental desde otra perspectiva, fuera del modelo clásico de atención hospitalaria hacia uno, centrado en la comunidad; pero aún queda un largo camino por recorrer ${ }^{(31)}$.

\section{Líneas de acción clave según la OPS/OMS}

Ante todo esto, en el año 2016 la OPS/OMS desarrolló una Guía Técnica de Salud Mental en Situaciones de Desastres y Emergencias ${ }^{(32)}$, en la cual se plantearon nueve líneas de acción para la preservación de la salud mental en las personas víctimas de desastres:

1) Asegurar que las intervenciones se desarrollen de acuerdo a las necesidades y recursos identificados.

2) Fortalecer las capacidades de los miembros de la comunidad e incluir a personas marginadas, a través de los mecanismos de autoayuda y el apoyo mutuo.
3) Garantizar que los trabajadores de ayuda humanitaria, agentes comunitarios, equipos de respuesta, así como el personal de los servicios de salud, estén capacitados y ofrezcan la primera ayuda psicológica a las víctimas y personas con un elevado grado de sufrimiento luego de una exposición a un desastre.

4) Asegurar que los equipos de salud que actúen como primera línea de contacto con la población dispongan de la capacidad para identificar y manejar los problemas psicosociales y de salud mental más frecuentes (TEPT, trastornos depresivos, consumo perjudicial de sustancias); así como que los servicios especializados se fortalezcan y se acerquen a la comunidad.

5) Gestionar y apoyar las medidas de seguridad; así como aquellas orientadas a satisfacer las necesidades y derechos básicos de las personas con trastornos mentales que se encuentren internadas en diferentes 
instituciones.

6) Minimizar el daño relacionado al alcohol y las drogas.

7) Iniciar los planes para desarrollar un sistema de salud comunitario sostenible.

8) Contribuir a una apropiada comunicación de riesgo y gestión de la información en la respuesta a emergencia y desastres.

9) Garantizar la atención priorizada a condiciones identificadas de alto riesgo psicosocial como miembros del equipo de emergencia, personas adultas mayores o con algún trastorno mental severo y población en extrema pobreza.

En conclusión, los desastres son situaciones, casi siempre imprevistas, que ocasionan un gran daño material o humano, que suele traer consecuencias en la estructura social. Usualmente, no existen suficientes recursos materiales ni sociales para afrontarlo, y su gravedad radicará en las consecuencias, más que en las causas mismas. Existe una gran variabilidad en la respuesta psicosocial a los desastres, y esta dependerá no solo de la naturaleza del desastre ni del ambiente y las circunstancias asociadas, sino también, de las características de las personas que lo experimentan. Algunos de los factores de vulnerabilidad asociadas al desarrollo de un trastorno mental son el antecedente de una alteración mental previa, ser del género femenino, los menores de edad, pertenecer a un grupo étnico minoritario y pobre apoyo social.

Todo esto podría generar diversos problemas de salud mental, como el TEPT, la depresión y el consumo de múltiples sustancias, que son los más frecuentes. Es necesario que, como país, tengamos diversas estrategias y modelos de intervención para intentar atenuar el impacto psicosocial del desastre. Sin embargo, aún existen barreras en el manejo de la salud mental por lo que se hace necesario plantear posibles direcciones futuras, a fin de lograr un menor impacto en la sociedad y una recuperación más temprana.

\section{REFERENCIAS BIBLIOGRÁFICAS}

1. North CS. Disaster Mental Health Epidemiology: Methodological Review and Interpretation of Research Findings. Psychiatry. 2016;79(2):130-46.

2. Bambarén $C$. Salud mental en desastres naturales. Rev Psicol Hered. 2011;6(1-2):20-5.

3. Diccionario etimológico español [Internet]. Desastre [citado 9 de enero de 2019]. Recuperado a partir de: http:// etimologias.dechile.net/?desastre.

4. Calderón J. La salud mental en los desastres. Bogotá: Cruz Roja Colombiana; 2002.

5. Goldmann E, Galea S. Mental health consequences of disasters. Annu Rev Public Health. 2014;35:169-83.
6. Math SB, Nirmala MC, Moirangthem S, Kumar NC. Disaster Management: Mental Health Perspective. Indian J Psychol Med. 2015;37(3):261-71.

7. Villalibre Calderón C. Concepto de urgencia, emergencia y desastre: Revisión histórica y bibliográfica [Master en análisis y gestión de emergencia y desastre]. Oviedo: Universidad de Oviedo; 2013.

8. El Comercio. COEN: a 145 creció número de muertos por lluvias e inundaciones. [Internet]. Lima: El Comercio; 2017 [citado 11 de enero de 2019]. Recuperado a partir de:https:// elcomercio.pe/peru/coen-145-crecio-numero-muertoslluvias-e-inundaciones-424733.

9. Ball P, Asher J, Sulmont D, Manrique D. ¿Cuántos peruanos murieron? Estimación del total de víctimas causadas por el conflicto armado interno entre 1980 y el 2000. Lima: Comisión de la Verdad y Reconciliación; 2003: 52.

10. Southwick SM, Satodiya R, Pietrzak RH. Disaster Mental Health and Positive Psychology: An Afterward to the Special Issue. J Clin Psychol. 2016;72(12):1364-68.

11. McCabe OL, Kaminsky MJ, McHugh PR. Clinical assessment in disaster mental health: a logic of case formulation. Am J Disaster Med. 2007;2(6):297-306.

12. Reifels L, Pietrantoni L, Prati G, Kim Y, Kilpatrick DG, Dyb $\mathrm{G}$, et al. Lessons learned about psychosocial responses to disaster and mass trauma: an international perspective. Eur J Psychotraumatol. 2013;4:1-9.

13. Abeldano RA, Fernandez R. Community Mental Health in disaster situations. A review of community-based models of approach. Cien Saude Colet. 2016;21(2):431-42.

14. North CS, Pfefferbaum B. Mental health response to community disasters: a systematic review. Jama. 2013;310(5):507-18.

15. Galea S, Nandi A, Vlahov D. The epidemiology of posttraumatic stress disorder after disasters. Epidemiol Rev. 2005;27:78-91.

16. Neria Y, Nandi A, Galea S. Post-traumatic stress disorder following disasters: a systematic review. Psychol Med. 2008;38(4):467-80.

17. Norris FH, Friedman MJ, Watson PJ, Byrne CM, Diaz E, Kaniasty K. 60,000 disaster victims speak: Part I. An empirical review of the empirical literature, 1981-2001. Psychiatry. 2002;65(3):207-39.

18. Bell SA, Folkerth LA. Women's Mental Health and Intimate Partner Violence Following Natural Disaster: A Scoping Review. Prehosp Disaster Med. 2016;31(6):648-57.

19. Parker G, Lie D, Siskind DJ, Martin-Khan M, Raphael B, Crompton D, et al. Mental health implications for older adults after natural disasters--a systematic review and metaanalysis. Int Psychogeriatr. 2016;28(1):11-20.

20. Fernandez A, Black J, Jones $M$, Wilson L, Salvador-Carulla $L$, Astell-Burt T, et al. Flooding and mental health: a systematic mapping review. PLoS One. 2015;10(4):e0119929.

21. Contreras C, Aguilar M, Eappen B, Guzman C, Carrasco P, Millones AK, et al. Community strengthening and mental health system linking after flooding in two informal human settlements in Peru: a model for small-scale disaster response. Glob Ment Health (Camb). 2018;5:e11.

22. Cobham VE, McDermott B, Haslam D, Sanders MR. The Role of Parents, Parenting and the Family Environment in Children's Post-Disaster Mental Health. Curr Psychiatry Rep. 2016;18(6):53.

23. Williamson V, Creswell C, Butler I, Christie H, Halligan S. 
Parental experiences of supporting children with clinically significant post-traumatic distress: a Qualitative study of families accessing psychological services. Journ child adol trauma. 2017:1-12.

24. Pfefferbaum B, Jacobs AK, Houston JB, Griffin N. Children's disaster reactions: the influence of family and social factors. Current psychiatry reports. 2015;17(7):57.

25. Maclean JC, Popovici I, French MT. Are natural disasters in early childhood associated with mental health and substance use disorders as an adult? Soc Sci Med. 2016;151:78-91.

26. Lowe SR, Rhodes JE, Scoglio AA. Changes in Marital and partner relationships in the aftermath of hurricane katrina: an analysis with low-Income women. Psychol women Q. 2012;36(3):286-300.

27. Perú21. Evangelina Chamorro: El símbolo de lucha en medio de la tragedia. [Internet]. Lima: Perú21; 2017 [citado 11 de enero de 2019].Recuperado a partir de:https://peru21.pe/ peru/tragedias-2017-evangelina-simbolo-lucha-390021.

28. OPS/OMS. Emergencia por impacto del Fenómeno "El niño costero" - Perú. [Internet]. 2017 [citado 11 de enero de 2019]. Recuperado a partir de:https://peru21.pe/peru/tragedias2017-evangelina-simbolo-lucha-390021 https://www.paho. org/per/index.php?option=com_content\&view=article\&id=3 710:emergencia-por-impacto-del-fenomeno-el-nino-costero2017-peru\&Itemid=1060.

29. Steering Committee for Humanitarian Response (SCHR) VOiCiEV, International Council of Voluntary Agencies (ICVA). Proyecto Esfera: Carta humanitaria y normas mínimas de respuesta humanitaria en casos de desastre. Ginebra. 2011.

30. Kazlauskas E. Challenges for providing health care in traumatized populations: barriers for PTSD treatments and the need for new developments. Glob Health Action. 2017;10(1):1322399.

31. Fraser B. Earthquake highlights mental-health issues in Peru. Lancet. 2007;370(9590):815-6.
32. Organización Panamericana de la Salud. Guía técnica de salud mental en situaciones de desastres y emergencias 2016 [Internet]. [citado 10 de enero de 2019]. Recuperado a partir de:https: / / www.paho.org/disasters/index.php?option=com docman\&view=download\&category_slug=books\&alias=2443guia-tecnica-salud-mental-situaciones-desastresemergencias $-3 \&$ Itemid=1179\&lang=es.

Fuentes de financiamiento:

Este artículo ha sido financiado por los autores.

Conflictos de interés:

Los autores declaran no tener ningún conflicto de interés.

\section{Correspondencia:}

Jeff David Huarcaya Victoria

Dirección: Av. Alameda del Corregidor, 1531, La Molina. Lima, Perú.

Teléfono: 511-3653640

Correo electrónico: jhuarcayav@usmp.pe

\section{Recibido: 18 de enero de 2019 \\ Evaluado: 25 de enero de 2019 \\ Aprobado: 05 de febrero de 2019}

( ) La revista. Publicado por Universidad de San Martín de Porres, Perú. (cc) Br Licencia de Creative Commons Artículo en acceso abierto bajo términos de Licencia Creative Commons Atribución 4.0 Internacional. (http://creativecommons.org/licenses/by/4.0/)

\section{ORCID iDs}

Deborah León Amenero

Jeff Huarcaya Victoria
https://orcid.org/0000-0001-7359-723X https://orcid.org/0000-0003-4525-9545 